OPEN

SUBJECT AREAS:

EXPERIMENTAL PARTICLE

PHYSICS

SPINTRONICS

Received

5 March 2014

Accepted

10 April 2014

Published

29 April 2014

Correspondence and requests for materials should be addressed to H.J.Z. (zhang. hongjun@jaea.go.jp)

\section{Current-induced spin polarization on metal surfaces probed by spin-polarized positron beam}

\author{
H. J. Zhang' ' S. Yamamoto' ${ }^{2}$, Y. Fukaya' ', M. Maekawa' , H. Li' , A. Kawasuso', T. Seki' ${ }^{3}$ E. Saitoh ${ }^{3}$ \\ \& K. Takanashi ${ }^{3}$
}

1Advanced Science Research Center, Japan Atomic Energy Agency, 1233 Watanuki, Takasaki, Gunma 370-1292, Japan,
${ }^{2}$ Quantum Beam Science Directorate, Japan Atomic Energy Agency, 1233 Watanuki, Takasaki, Gunma 370-1292, Japan,
${ }^{3}$ Institute for Materials Research, Tohoku University, 2-1-1 Katahira, Aoba-ku, Sendai 980-8577, Japan.

Current-induced spin polarization (CISP) on the outermost surfaces of $\mathrm{Au}, \mathrm{Cu}, \mathrm{Pt}, \mathrm{Pd}, \mathrm{Ta}$, and $\mathrm{W}$ nanoscaled films were studied using a spin-polarized positron beam. The Au and $\mathrm{Cu}$ surfaces showed no significant CISP. In contrast, the Pt, Pd, Ta, and W films exhibited large CISP (3 $\sim 15 \%$ per input charge current of $10^{5} \mathrm{~A} / \mathrm{cm}^{2}$ ) and the CISP of Ta and W were opposite to those of Pt and Pd. The sign of the CISP obeys the same rule in spin Hall effect suggesting that the spin-orbit coupling is mainly responsible for the CISP. The magnitude of the CISP is explained by the Rashba-Edelstein mechanism rather than the diffusive spin Hall effect. This settles a controversy, that which of these two mechanisms dominates the large CISP on metal surfaces.

pintronics, which aims to produce, inject, transport, manipulate, and detect the electron spins, is promising to go beyond the traditional charge-based electronics ${ }^{1}$. Current-induced spin polarization (CISP) plays a critical role in spintronics. The spin Hall effect (SHE) and the Rashba effect are the representative phenomena producing CISP.

In the SHE, a finite spin current appears due to the charge current and the spin-orbit coupling (SOC). The efficiency of charge-to-spin conversion is defined as the ratio of spin to charge current densities $\left(\theta_{S H}=j_{s} / j_{c}\right.$, called spin Hall angle). At sample edges, opposite electron spins are accumulated. Large spin Hall effects have been found in metallic thin films of $\mathrm{Pt}^{2}, \mathrm{Pd}^{3}, \beta-\mathrm{Ta}^{4}$, and $\beta-\mathrm{W}^{5}$.

The Rashba effect induces in-plane spin polarization in a two-dimensional electron gas (2DEG) system through the out-of-plane electric field and the SOC 6 . The strength of the Rashba effect is characterized by the energy splitting of up and down spin bands. The Rashba splitting energy observed for semiconductor heterostructure is only a few meV. Recently, so-called giant Rashba effects (of the order of $100 \mathrm{meV}$ ) have been reported for bulk $\operatorname{Ir}(111)^{7}, \mathrm{Bi} / \mathrm{Ag}(111)$ surface alloy ${ }^{8}$, and $\mathrm{Pb} / \operatorname{Ag}(111)$ surface alloy ${ }^{9}$.

It is still under debate that, which of the above two mechanisms is responsible for the large CISP on metal surfaces and interfaces. To solve this issue, surface sensitive probes are needed. Magneto-optical Kerr effect magnetometry is used for the observation of SHE in semiconductors ${ }^{10}$. However, this technique is not applicable to metallic thin films with thickness ranging from several $\mathrm{nm}$ to a few tens of $\mathrm{nm}$. Spin-polarized positron beam is a promising new tool for such a purpose. Positronium (Ps), which is a bound state of a positron and an electron, is formed at the outermost surface of a metal ${ }^{11}$. From the spin-dependence of Ps formation and annihilation, the spin polarization of metal surface can be determined ${ }^{12}$. Recently, we reported the observation of the CISP on Pt surfaces ${ }^{13}$ by this technique. However, the origin of the observed CISP was not clarified. In the present study, we systematically investigate CISP in some other $4 d$ and $5 d$ transition metals. Consequently, we found that the CISP on these metal surfaces is explained in terms of the Rashba-Edelstein mechanism.

\section{Results}

Experimental setup and the principle. Figure 1 shows a schematic diagram of the experimental setup. The transversely spin-polarized positron beam, which was generated by a ${ }^{22} \mathrm{Na}$ source $(370 \mathrm{MBq})$ and an electrostatic apparatus, was implanted into the center of the sample ${ }^{14}$. The diameter and the spin polarization $\left(P_{+}\right)$of the positron beam were $1 \mathrm{~mm}$ and 0.3 , respectively. The beam energy $\left(E_{+}\right)$was adjusted from $50 \mathrm{eV}$ to $12 \mathrm{keV}$. The 


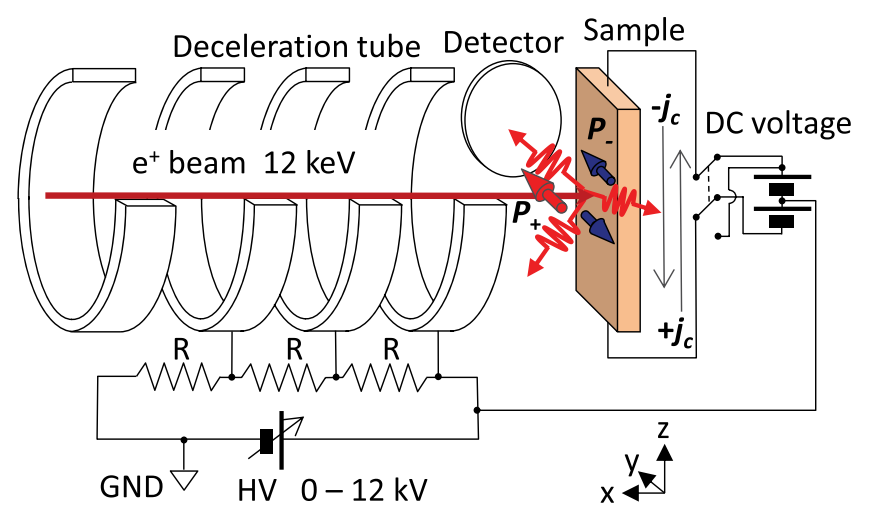

Figure $1 \mid$ Experimental setup. Transversely polarized positrons are injected into the center of the sample under a direct current $\left( \pm j_{c}\right)$. The beam energy of $12 \mathrm{keV}$ is reduced to $50 \mathrm{eV}$ by a deceleration tube. The $\gamma$ ray detector is perpendicular to the beam axis.

sample center was electrically grounded. Reversible currents $\left( \pm j_{c}\right)$ were applied to the samples through the two edges. The direct current was perpendicular to $P_{+}$. A high purity Ge detector was placed perpendicular to the beam axis to record the annihilation $\gamma$ ray spectra.

Spin-polarized slow (low energy) positrons injected into a metallic thin film could lead to a remarkable formation of Ps by picking up the electrons on the outermost surface. The formation probability of ortho-Ps $\left(F_{P s}^{3 \gamma}\right)$, which is influenced by the spin polarization of the outermost surface electrons $\left(P_{-}\right)$, could be derived from the positron annihilation $\gamma$ ray spectra as the Ratio between the intensity of the low energy region and the $511 \mathrm{keV}$ peak region (denoted as $R$ ). A function $\Delta R$ is defined to quantitatively characterize the $F_{P S}^{3 \gamma}$ (details shown in the section of methods):

$$
\Delta R=R-R_{0},
$$

where $R$ and $R_{0}$ are derived from the $\gamma$ ray spectra measured at $E_{+}=50 \mathrm{eV}$ and $12 \mathrm{keV}$, respectively. The component of surface spin polarization along $y$ axis $\left(P_{-} \cos \phi\right)$ is calculated by

$$
P_{-} \cos \phi=\frac{\Delta R_{+j_{c}}-\Delta R_{-j_{c}}}{0.18\left(\Delta R_{+j_{c}}+\Delta R_{-j_{c}}\right)},
$$

where $\phi$ is the relative angle of $P_{-}$to $P_{+}$( $y$ axis), $\Delta R_{+j_{c}}$ and $\Delta R_{-j_{c}}$ correspond to an input charge current density of $+j_{c}$ and $-j_{c}$, respectively.

Experiments. All films were deposited by magnetron sputtering on different substrates $(10 \times 20 \times 0.5 \mathrm{~mm})$ at various growth temperatures. The details of the films are listed in Table 1. The thickness of Fe seed layer for Au film was $1 \mathrm{~nm}$. The low resistivity ( $\alpha$ phase: bcc structure) Ta and $\mathrm{W}$ films were grown on $\mathrm{Al}_{2} \mathrm{O}_{3}(0001)$ substrates, and the high resistivity ( $\beta$ phase: A15 structure) Ta and W

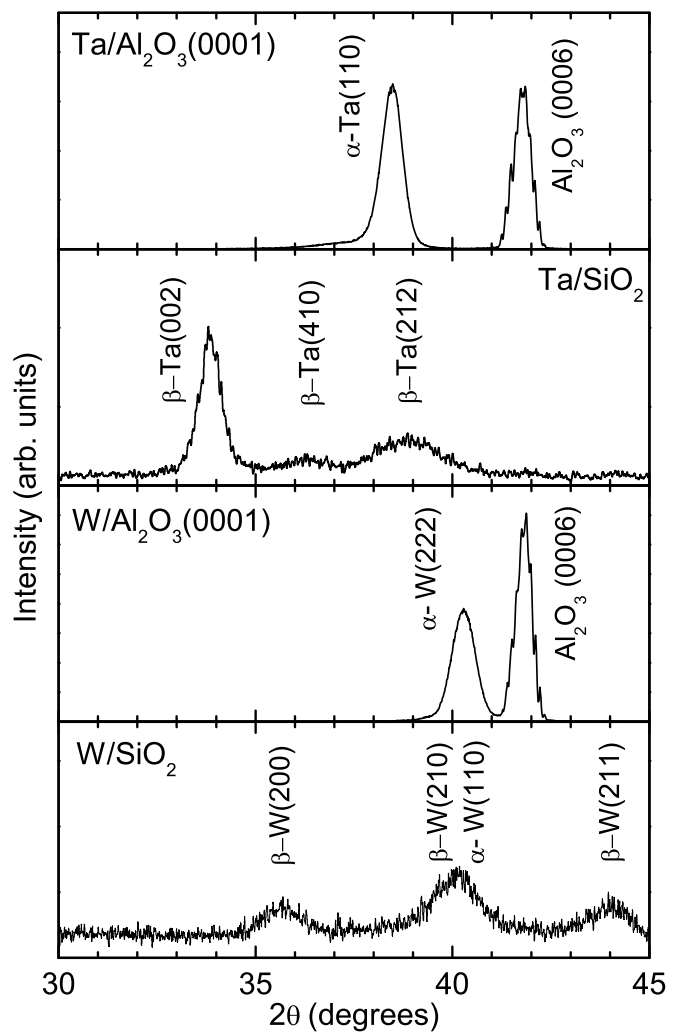

Figure $2 \mid \mathrm{XRD}$ patterns of $\alpha$ - $\mathrm{Ta} / \mathrm{Al}_{2} \mathrm{O}_{3}(0001), \boldsymbol{\beta}-\mathrm{Ta} / \mathrm{SiO}_{2} / \mathrm{Si}(001), \alpha$-W/ $\mathrm{Al}_{2} \mathrm{O}_{3}(0001)$, and $\beta \alpha-\mathrm{W} / \mathrm{SiO}_{2} / \mathrm{Si}(001)$ samples.

films were grown on $100 \mathrm{~nm}$ thick $\mathrm{SiO}_{2}$ layers. The $\mathrm{Au}, \mathrm{Pt}, \mathrm{Pd}, \alpha-\mathrm{Ta}$, and $\alpha-\mathrm{W}$ films were single crystals, which were confirmed by observing the reflection high energy electron diffraction patterns. The $\mathrm{Cu}, \beta$-Ta, and $\beta \alpha$-W (a mixture of $\beta$ and $\alpha$ phases in which $\beta$ is dominant) films were polycrystals. The XRD patterns shown in Fig. 2 confirmed the $\alpha$-Ta, $\beta$-Ta, $\alpha$-W, and $\beta \alpha$-W films ${ }^{15-17}$. At least two samples were subjected to the CISP measurement for each film.

Input charge current densities $j_{c}$ are also listed in Table 1. To suppress the Joule heating, the applied electric powers were regulated to be less than 3 watts and the temperature was measured to be lower than $150^{\circ} \mathrm{C}$. In this temperature range, fast Ps with the maximum energy of its work function $\left(\Phi_{P_{s}} \approx 0.7 \mathrm{eV}(\mathrm{Au}), 2.5 \mathrm{eV}(\mathrm{Cu}), 2.9 \mathrm{eV}\right.$ (Pt), $0.4 \mathrm{eV}(\mathrm{Pd}), 4.0 \mathrm{eV}(\mathrm{Ta}(111)), 4.9 \mathrm{eV}(\mathrm{W}(111)))$ will be predominant over the thermal $(\sim 100 \mathrm{meV}) \mathrm{Ps}^{18-21}$. Therefore, positrons will pick up surface electrons with the energy from $E_{F}($ Fermi level) to $E_{F}-\Phi_{P s}$.

Material dependence of CISP. Figure 3 shows $\Delta R\left(\propto F_{P s}^{3 \gamma}\right)$ upon successive current reversal $\left(+j_{c} \leftrightarrow-j_{c}\right)$ of all the films. For the Au and $\mathrm{Cu}$ films, no regular changes of $\Delta R$ upon current reversal could

Table 1 | Sample characteristics (film thickness $\left(t_{N}\right)$, substrate, growth temperature $\left(T_{g}\right)$, resistivity $\left.(\rho)\right)$, input charge current density $\left(i_{c}\right)$ and

\begin{tabular}{|c|c|c|c|c|c|c|}
\hline Sample & $t_{N}(\mathrm{~nm})$ & Substrate & $\mathrm{T}_{g}\left({ }^{\circ} \mathrm{C}\right)$ & $\rho(\mu \Omega \mathrm{cm})$ & $i_{c}\left(\mathrm{~A} / \mathrm{cm}^{2}\right)$ & $P_{-} \cos \phi(\%)$ \\
\hline $\mathrm{Au}(001)$ & 25 & $\mathrm{Fe}(001) / \mathrm{MgO}(001)$ & 27 & 16 & $2.4 \times 10^{5}$ & Null \\
\hline $\mathrm{Cu}$ & 25 & $\mathrm{MgO}(001)$ & 27 & 8 & $2.4 \times 10^{5}$ & Null \\
\hline $\operatorname{Pt}\left(\begin{array}{lll}1 & 1 & 1\end{array}\right)$ & 25 & $\mathrm{Al}_{2} \mathrm{O}_{3}(0001)$ & 600 & 21 & $2.0 \times 10^{5}$ & $11 \pm 2$ \\
\hline$\alpha-\mathrm{Ta}$ & 10 & $\mathrm{Al}_{2} \mathrm{O}_{3}(0001)$ & 600 & 43 & $2.5 \times 10^{5}$ & $-12 \pm 3$ \\
\hline$\beta-\mathrm{Ta}$ & 10 & $\mathrm{SiO}_{2} / \mathrm{Si}(001)$ & 27 & 128 & $5.0 \times 10^{4}$ & $-7 \pm 2$ \\
\hline$\alpha-W$ & 10 & $\mathrm{Al}_{2} \mathrm{O}_{3}(0001)$ & 600 & 28 & $1.0 \times 10^{5}$ & $-6 \pm 2$ \\
\hline
\end{tabular}
observed transverse spin polarization $\left(P_{-} \cos \phi\right)$ 


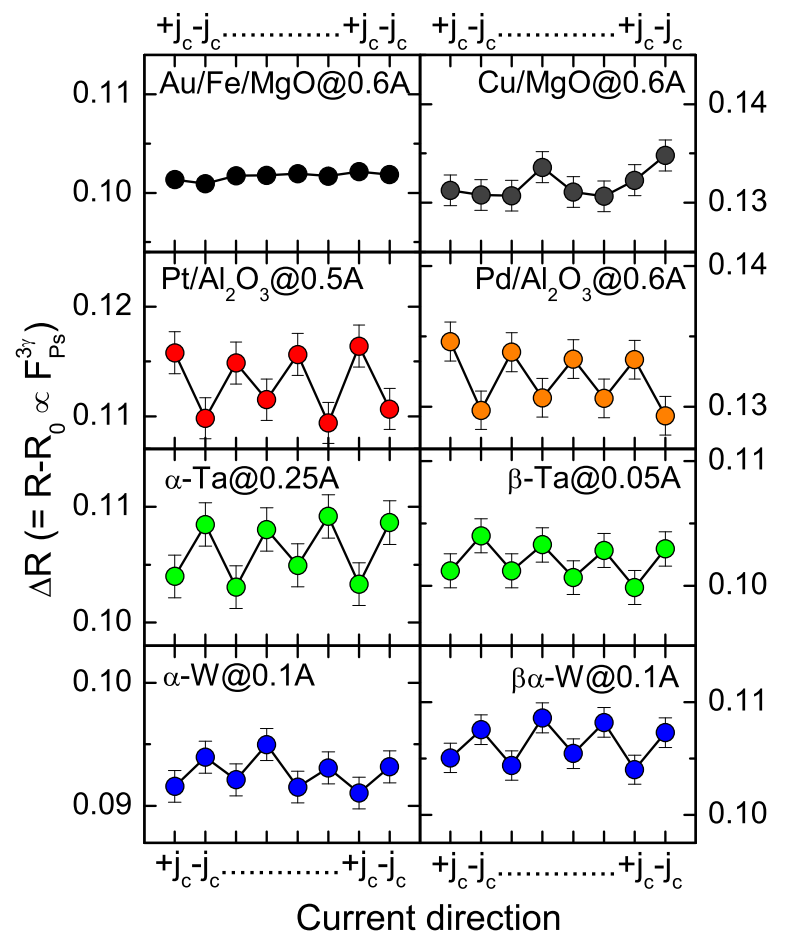

Figure $3 \mid \Delta R$ as a function of successive current reversals for the $\mathrm{Au}(001) / \mathrm{Fe}(001) / \mathrm{MgO}(001), \mathrm{Cu} / \mathrm{MgO}(001), \mathrm{Pt}(111) / \mathrm{Al}_{2} \mathrm{O}_{3}(0001)$, $\mathrm{Pd}(111) / \mathrm{Al}_{2} \mathrm{O}_{3}(0001), \alpha-\mathrm{Ta} / \mathrm{Al}_{2} \mathrm{O}_{3}(0001), \beta-\mathrm{Ta} / \mathrm{SiO}_{2} / \mathrm{Si}(001), \alpha-\mathrm{W} /$ $\mathrm{Al}_{2} \mathrm{O}_{3}(0001)$ and $\beta \alpha-\mathrm{W} / \mathrm{SiO}_{2} / \mathrm{Si}(001)$ samples.

be seen. In contrast, the $\mathrm{Pt}, \mathrm{Pd}$, Ta and $\mathrm{W}$ surfaces show clear oscillations of $\Delta R$. In addition to this, the $\Delta R$ oscillations of Ta and $\mathrm{W}$ films are opposite to those of $\mathrm{Pt}$ and $\mathrm{Pd}$ films. These results suggest that the CISP on the $\mathrm{Au}$ and $\mathrm{Cu}$ surfaces are rather small $\left(P_{-} \cos \phi \ll 1 \%\right)$, while significant CISP are induced on the Pt, Pd, $\mathrm{Ta}$, and $\mathrm{W}$ surfaces. Also, the CISP on Ta and $\mathrm{W}$ surfaces are opposite to those on $\mathrm{Pt}$ and $\mathrm{Pd}$ surfaces. The transverse spin polarizations $\left(P_{-} \cos \phi\right)$ estimated by Eq. $(2)$ are listed in Table 1.

Figure 4 shows $P_{-} \cos \phi$ per input charge current of $j_{c}=1.0 \times$ $10^{5} \mathrm{~A} / \mathrm{cm}^{2}$. The absolute values of $P_{-} \cos \phi$ for the $\beta$-Ta and $\beta \alpha-\mathrm{W}$ surfaces are $3 \sim 5$ times greater than those for the Pt and Pd surfaces.

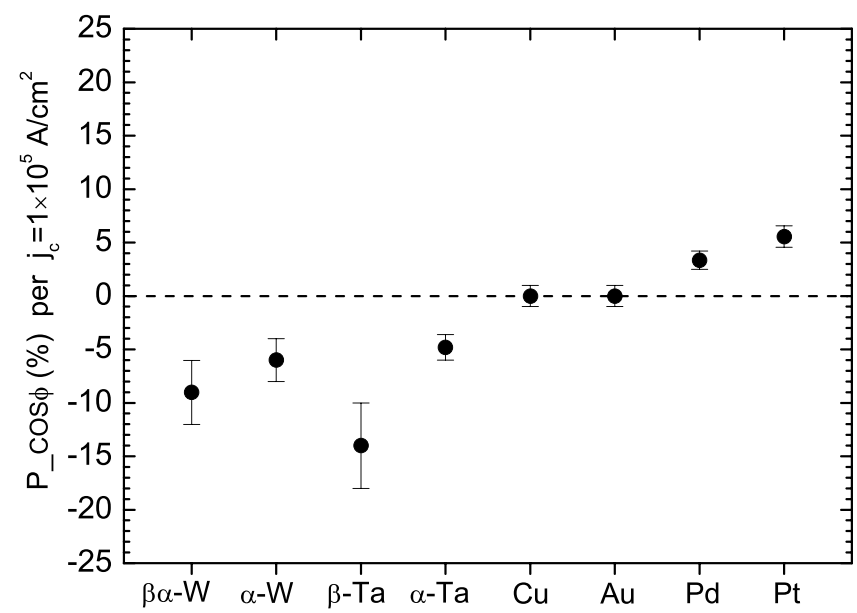

Figure $4 \mid$ Spin polarizations of surface electrons $\left(P_{-} \cos \phi\right)$ per input charge current of $j_{c}=1 \times 10^{5} \mathrm{~A} / \mathrm{cm}^{2}$ for the $\mathrm{Au}(001) / \mathrm{Fe}(001) / \mathrm{MgO}(001)$, $\mathrm{Cu} / \mathrm{MgO}(001), \mathrm{Pt}(111) / \mathrm{Al}_{2} \mathrm{O}_{3}(0001), \mathrm{Pd}(111) / \mathrm{Al}_{2} \mathrm{O}_{3}(0001), \alpha-\mathrm{Ta} /$ $\mathrm{Al}_{2} \mathrm{O}_{3}(0001), \beta-\mathrm{Ta} / \mathrm{SiO}_{2} / \mathrm{Si}(001), \alpha-\mathrm{W} / \mathrm{Al}_{2} \mathrm{O}_{3}(0001)$, and $\beta \alpha-\mathrm{W} / \mathrm{SiO}_{2} /$ $\mathrm{Si}(001)$ samples.
Table $2 \mid \theta_{S H}$ found by different experimental methods. YIG, CFB, SA, STT, SP, ISHE, ST-FMR, and SMR denote $\mathrm{Y}_{3} \mathrm{Fe}_{5} \mathrm{O}_{12}$, $\mathrm{Co}_{40} \mathrm{Fe}_{40} \mathrm{~B}_{20}$, spin absorption, spin transfer torque, spin pumping, inverse SHE, spin torque induced ferromagnetic resonance, and spin Hall magnetoresistance, respectively

\begin{tabular}{|c|c|c|c|}
\hline Film (nm) & $\theta_{S H}(\%)$ & Method & Ref. \\
\hline $\mathrm{Pt}(4) / \mathrm{Cu}(80)$ & 0.37 & SA & [22] \\
\hline $\mathrm{Pt}(10) / \mathrm{Py}(10)$ & 8.0 & STT & [23] \\
\hline $\mathrm{Pt}(15) / \mathrm{Py}(15)$ & $1.3 \pm 0.2$ & $\mathrm{SP} / \mathrm{ISHE}$ & [24] \\
\hline $\operatorname{Pt}(6) / \operatorname{Py}(4)$ & $7.6 \pm_{2.6}^{8.4}$ & ST-FMR & [25] \\
\hline $\mathrm{Pt}(20) / \mathrm{Cu}(150)$ & $2.1 \pm 0.5$ & SA & [26] \\
\hline $\mathrm{Pt}(2-9) / \mathrm{Py}(2.7-10.5)$ & $2.2 \pm 0.4$ & ST-FMR & [27] \\
\hline $\mathrm{Pt}(15) / \mathrm{YIG}$ & $3.0 \pm \pm_{1.5}^{4.0}$ & SMR & [28] \\
\hline $\mathrm{Pt}(1.1-22.7) / \mathrm{YIG}$ & $11 \pm 8$ & SMR & [29] \\
\hline $\mathrm{Pd}(10) / P y(10)$ & 1.0 & $\mathrm{SP}$ & [3] \\
\hline $\mathrm{Pd}(15) / \mathrm{Py}(15)$ & $0.64 \pm 0.10$ & SP/ISHE & [24] \\
\hline $\mathrm{Pd}(20) / \mathrm{Cu}(150)$ & $1.2 \pm 0.4$ & SA & [26] \\
\hline $\operatorname{Pd}(2-9) / \operatorname{Py}(2.2-7.0)$ & $0.8 \pm 0.2$ & ST-FMR & [27] \\
\hline $\mathrm{Au}(15) / \mathrm{Py}(15)$ & $0.35 \pm 0.03$ & $\mathrm{SP} / \mathrm{ISHE}$ & [24] \\
\hline $\mathrm{Ta}(20) / \mathrm{Cu}(150)$ & $-0.37 \pm 0.11$ & SA & [26] \\
\hline$\beta$-Ta(4)/CFB(4) & $-12 \pm 3$ & ST-FMR & {$[4]$} \\
\hline$\beta$-Ta(8)/CFB(4) & $-15 \pm 3$ & ST-FMR & [4] \\
\hline $\mathrm{Ta}(1.5-15) / \mathrm{YIG}$ & $-2.0 \pm_{1.5}^{0.8}$ & SMR & [28] \\
\hline$\beta$-W(5.2)/CFB(4) & $-33 \pm 6$ & ST-FMR & {$[5]$} \\
\hline$(\alpha+\beta)-W(6.2) / C F B(4)$ & $-18 \pm 2$ & ST-FMR & [5] \\
\hline$\alpha-W(15) / C F B(4)$ & $>-7$ & ST-FMR & [5] \\
\hline
\end{tabular}

For both high resistivity Ta and $\mathrm{W}$ films, $P_{-} \cos \phi$ are significantly bigger than those of low resistivity Ta and $\mathrm{W}$ films.

\section{Discussion}

Table 2 lists the $\theta_{S H}$ of undoped metals obtained by different experimental methods. The values of $\theta_{S H}$ are rather scattered. Even for Pt, which is the most commonly studied spin Hall material, $\theta_{S H}$ varies between $0.37 \%$ and $11.0 \%$. The $\mathrm{Pt}, \mathrm{Pd}$, and $\mathrm{Au}$ films have positive $\theta_{S H}$, while the $\mathrm{Ta}$ and $\mathrm{W}$ films have negative $\theta_{S H}$. Furthermore, absolute values of $\theta_{S H}$ of Ta and $\mathrm{W}$ tend to be greater than those of $\mathrm{Pt}$ and $\mathrm{Pd}$. The magnitudes of $\theta_{S H}$ of $\beta$ phase Ta and $\mathrm{W}$ films have been reported to be much bigger than those in $\alpha$ phases $^{4,5}$. These observations of $\theta_{S H}$ are mostly supported by theoretical studies of $\theta_{S H}$ in which the sign is positive (negative) if the outermost $d$-shell is more (less) than half filling ${ }^{30,31}$.

The sign and relative magnitude of the CISP observed for the Pt, $\mathrm{Pd}, \mathrm{Ta}$, and $\mathrm{W}$ surfaces are in good agreement with those of $\theta_{S H}$ listed in Table 2. This reveals that the observed CISP for these surfaces are due to the SOC that is similar to SHE. According to the spin diffusion theory ${ }^{32}$, the energy width of polarized electrons in the density of states is given by the shift of chemical potential: $\Delta \mu=2 \theta_{S H} \lambda_{S} j_{c} \rho$, where $\lambda_{S}$ is the spin diffusion length. For $\theta_{S H}=10 \%, \lambda_{S}=10 \mathrm{~nm}$, $\rho=50 \mu \Omega \mathrm{cm}$ and $j_{c}=1.0 \times 10^{5} \mathrm{~A} / \mathrm{cm}^{2}$, one finds $\Delta \mu=1 \mu \mathrm{eV}$. The typical density of states at $E_{F}$ is $10^{23} \mathrm{~cm}^{-3} \mathrm{eV}^{-1}$, and hence the accumulated spin density will be $10^{17} \mathrm{~cm}^{-3}$. Assuming that positrons pick up electrons located from $E_{F}$ to $E_{F}-1 \mathrm{eV}$, the observable electron spin polarization will be $\sim 10^{-4} \%$. Therefore, the huge CISP observed above is hardly explained in terms of the diffusive SHE. More specific aspects of the surfaces should be considered.

Recently, the so-called giant Rashba effect has been reported for heavy metal surfaces ${ }^{7-9}$. The largest Rashba effects are five orders of magnitude greater than that estimated from the free electron model. Such a giant Rashba effect is explained by considering both strong SOC and steep gradient of electric potential near the surface. The spin density $\left\langle\delta s_{y}\right\rangle$ induced by the Rashba effect is given by

$$
\left\langle\delta s_{y}\right\rangle=4 \pi e D_{2 D} E \tau \alpha_{R} / \hbar,
$$

where $e$ is the elementary charge, $D_{2 D}$ is the two-dimensional density 
of states, $E$ is the applied electric field, $\tau$ is the electron relaxation time, and $\alpha_{R}$ is the Rashba parameter (Rashba-Edelstein model) ${ }^{33,34}$. Assuming $\alpha_{R}=3 \times 10^{-10} \mathrm{eVm}, D_{2 D}=10^{14} \mathrm{~cm}^{-2} \mathrm{eV}^{-1}, \tau=10 \mathrm{ps}$, $E=1 \mathrm{kV} / \mathrm{m}$, one finds the spin polarization of the order of $5 \%$. Thus, if the relaxation time is long enough, the above-observed huge CISP can be explained.

A recent study reported the spin-to-charge conversion at $\mathrm{Bi} / \mathrm{Ag}$ interface, which is a well-known giant Rashba system ${ }^{35}$. The spin density and the two-dimensional charge current density $j_{c}^{2 D}$ at an interface are related through $\left\langle\delta s_{y}\right\rangle=\hbar j_{c}^{2 D} /\left(e \alpha_{R}\right)$, which is essentially the same as Eq. (3). In the above study, excess spins of $\left\langle\delta s_{y}\right\rangle=2 \times$ $10^{7} \mathrm{~cm}^{-2}$ supplied to the $\mathrm{Bi} / \mathrm{Ag}$ interface by the spin pumping induced $j_{c}^{2 D}=10^{-5} \mathrm{~A} / \mathrm{cm}$. In the Ag layer, the spin-to-charge conversion was negligible and independent of its thicknesses (5 to $20 \mathrm{~nm}$ ). This would manifest that the spin-to-charge conversion was induced by an inverse Rashba effect but not inverse SHE. If we adopt this conversion efficiency in the present experiments, the twodimensional charge current density $j_{c}^{2 D}(0.05$ to $0.5 \mathrm{~A} / \mathrm{cm})$ will generate excess surface spins of $\left\langle\delta s_{y}\right\rangle=10^{12} \mathrm{~cm}^{-2}$ at maximum. Thus, assuming again $D_{2 D}=10^{14} \mathrm{~cm}^{-2} \mathrm{eV}^{-1}$, one finds the spin polarization of $1 \%$. This is comparable orders of magnitude as the above estimation using Eq. (3) in spite of many differences in experimental conditions. The $\alpha_{R}$ and $\theta_{S H}$ are related via $\left|\theta_{S H}\right|=m \alpha_{R}^{2} \tau^{36}$. This may be the reason why the sign and the relative magnitude of the CISP observed here are in good agreement with those of $\theta_{S H}$.

Furthermore, besides the Rashba effect at the outermost surface, one may naturally expect that the metal/substrate interface could also contribute to the spin polarization on the outermost surface. The thicknesses of the metallic films (10 and $25 \mathrm{~nm}$ ) are close to the spin diffusion lengths of the electron in these transition metals. A potential gradient also exists at the metal/substrate interface due to the difference of the metal and the substrate. In consideration of the Rashba effect at the metal/substrate interface, the transverse spin polarization calculated from Eq. (3) will increase and be more consistent with the experimental result from spin-polarized positron beam. To check this assumption in a future research, a metal/substrate interface with a strong Rashba effect is needed for the experiment.

It is known that Pt and Pd nano-structures nearly satisfy the Stoner criterion and hence ferromagnetic behavior appears ${ }^{37,38}$. This implies that ferromagnetic order will easily be induced in $\mathrm{Pt}$ and Pd surfaces. A recent anomalous Hall effect study of a $\mathrm{Pt} / \mathrm{Al}_{2} \mathrm{O}_{3}(00 \overline{1} 0)$ sample suggests that a magnetic moment of $\sim 10 \mu_{B}$ is induced by an applied electric field ${ }^{39}$. The Rashba field induced by the charge current may also contributes to the development of ferromagnetic order on the surface.

To summarize, we have observed huge CISP on the outermost surfaces of $\mathrm{Pt}, \mathrm{Pd}, \mathrm{Ta}$, and $\mathrm{W}$ thin films by using a spin-polarized positron beam. The sign and magnitude of the CISP on these metal surfaces are explained by the Rashba-Edelstein mechanism. This work demonstrates that the spin-polarized positron beam is a useful technique for observing the outermost surface spin polarization of spintronics materials.

\section{Methods}

Figure 5(a) shows the principle of Ps formation and annihilation. Spin-polarized positrons implanted into the sub-surface region are emitted into vacuum as Ps. Two types of Ps exist: spin-triplet ortho-Ps $(|S, m\rangle=|1,0\rangle,|1,1\rangle,|1,-1\rangle)$ and spin-singlet para-Ps $(|S, m\rangle=|0,0\rangle)$, where $S$ and $m$ are the total spin and the magnetic quantum number, respectively. Ortho-Ps decays into three $\gamma$ rays, giving rise to a continuous energy distribution from 0 to $511 \mathrm{keV}$. Para-Ps decays into two $\gamma$ rays of $\sim 511 \mathrm{keV}$, that overlaps with direct annihilation of positrons with electrons inside the sample. In the deep region of the metal, the probability of Ps formation is negligible. Shown as the shaded area in Fig. 5(b), the $3 \gamma$ annihilation of ortho-Ps (below $511 \mathrm{keV}$ peak) near the surface is clearly observable.
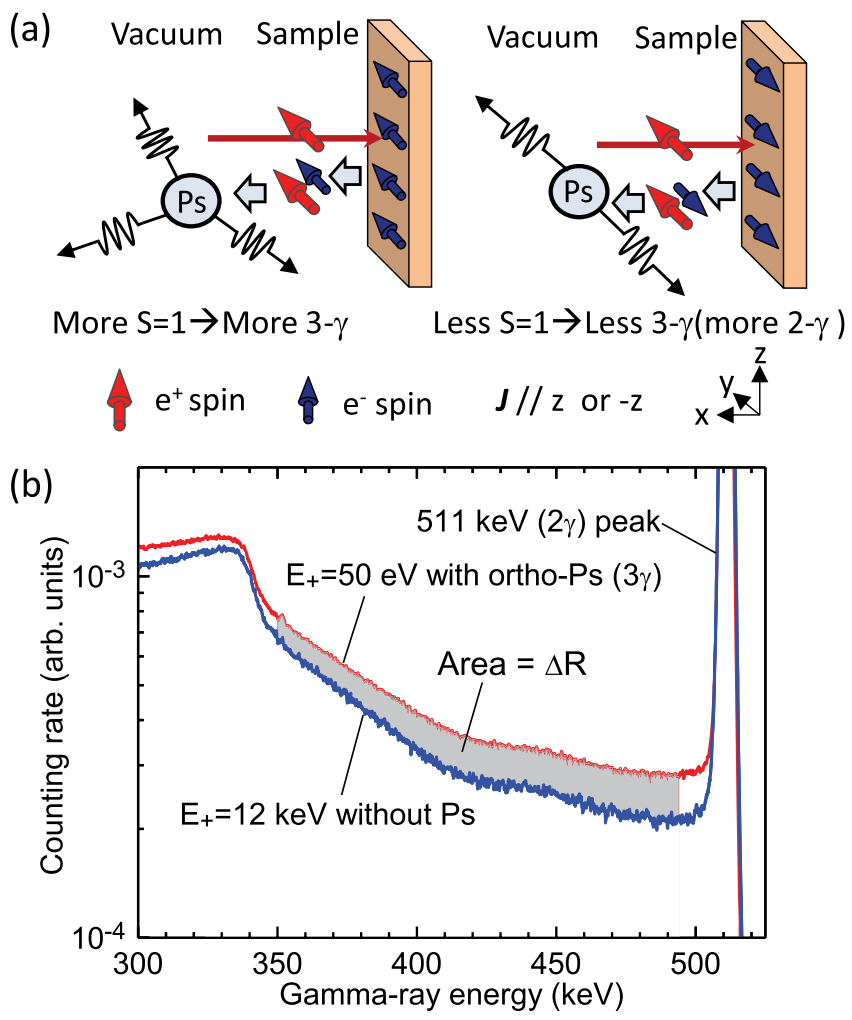

Figure $5 \mid$ (a) Principle of Ps formation and annihilation. Positrons $\left(\mathrm{e}^{+}\right)$ implanted into the subsurface region are emitted into vacuum as Ps by picking up electrons $\left(\mathrm{e}^{-}\right)$from the outermost surface. When the polarizations of positrons and electrons are parallel (anti-parallel), more (less) ortho-Ps $(\mathrm{S}=1)$ is formed. (b) Typical energy spectra of annihilation $\gamma$ rays obtained at positron energies of $E_{+}=12 \mathrm{keV}$ and $50 \mathrm{eV}$. The total intensity is normalized to the $511 \mathrm{keV}$ area intensity. The increment $\Delta \mathrm{R}$ represents the $3 \gamma$ annihilation of ortho-Ps.

The fraction of each spin state of Ps is given by ${ }^{13}$ :

$$
\begin{gathered}
F_{|0,0\rangle}=\left(1-P_{+} P_{-} \cos \phi\right) / 4, \\
F_{|1,0\rangle}=\left(1-P_{+} P_{-} \cos \phi\right) / 4, \\
F_{|1,1\rangle}=\left(1+P_{+}+P_{-} \cos \phi+P_{+} P_{-} \cos \phi\right) / 4, \\
F_{|1,-1\rangle}=\left(1-P_{+}-P_{-} \cos \phi+P_{+} P_{-} \cos \phi\right) / 4,
\end{gathered}
$$

where $P_{+}$and $P_{-}$are spin polarizations of the positrons and the electrons, respectively, and $\phi$ is the relative angle of $P_{-}$to $P_{+}$. The formation probability of para-Ps is $F_{P s}^{2 \gamma}=F_{|0,0\rangle}$, and that of ortho-Ps is

$$
F_{P s}^{3 \gamma}=\epsilon(1)\left(F_{|1,1\rangle}+F_{|1,-1\rangle}\right)+\epsilon(0) F_{|1,0\rangle},
$$

where $\epsilon(1)$ and $\epsilon(0)$ are detection efficiencies of annihilation $\gamma$ rays from $|1,1\rangle$ plus $\mid 1$, $-1\rangle$, and $|1,0\rangle$, respectively. The values of $\epsilon(1)$ and $\epsilon(0)$ depend on the angle between the $\gamma$ ray detector and $P_{+}$.

The intensity of the annihilation energy spectrum below $511 \mathrm{keV}$ is a function of $F_{P s}^{3 \gamma}$ :

$$
R=\frac{T-U}{U}=\frac{\left(1-F_{P_{s}}^{3 \gamma}\right) R_{0}+F_{P_{s}}^{3 \gamma} R_{1} U_{1} / U_{0}}{1-F_{P_{s}}^{3 \gamma}+F_{P_{s}}^{3 \gamma} U_{1} / U_{0}},
$$

where $T$ is the total area under the intensity curve, $U$ is the area under the $511 \mathrm{keV}$ peak, and the subscripts 0 and 1 of $R$ and $U$ denote $0 \%$ and $100 \%$ Ps emission, respectively. For small $F_{P_{s}}^{3 \gamma}, \Delta R=R-R_{0} \propto F_{P_{s}}^{3 \gamma}$. Thus, the asymmetry of $\Delta R$ upon spin flip ( $\left.+P_{-} \leftrightarrow-P_{-}\right)$ can be written as ${ }^{12}$

$$
\frac{\Delta R\left(+P_{-}\right)-\Delta R\left(-P_{-}\right)}{\Delta R\left(+P_{-}\right)+\Delta R\left(-P_{-}\right)}=\frac{2 \epsilon(1)-\epsilon(0)}{2 \epsilon(1)+\epsilon(0)} P_{+} P_{-} \cos \phi .
$$

From the known values of $P_{+}, \epsilon$, and the experimental asymmetry, the transverse spin polarization $\left(P_{-} \cos \phi\right)$ is determined. For the detector alignment in the present study 
(perpendicular to the positron beam), the factor $[2 \epsilon(1)-\epsilon(0)] /[2 \epsilon(1)+\epsilon(0)]$ in Eq. (10) is 0.6 .

1. Maekawa, S., Valenzuela, S. O., Saitoh, E. \& Kimura, T. Spin Current (Oxford University Press, Oxford, England, 2012).

2. Saitoh, E., Ueda, M., Miyajima, H. \& Tatara, G. Conversion of spin current into charge current at room temperature: Inverse spin Hall effect. Appl. Phys. Lett. 88, 182509 (2006).

3. Ando, K. \& Saitoh, E. Inverse spin Hall effect in palladium at room temperature. J. Appl. Phys. 108, 113925 (2010).

4. Liu, L. Q. et al. Spin-torque switching with the giant spin Hall effect of tantalum. Science 336, 555-558 (2012).

5. Pai, C. F. et al. Spin transfer torque devices utilizing the giant spin Hall effect of tungsten. Appl. Phys. Lett. 101, 122404 (2012).

6. Bychkov, Y. A. \& Rashba, E. I. Oscillatory effects and the magnetic susceptibility of carriers in inversion layers. J. Phys. C 17, 6039-6045 (1984).

7. Varykhalov, A. et al. $\operatorname{Ir}(111)$ surface state with giant Rashba splitting persists under graphene in air. Phys. Rev. Lett. 108, 066804 (2012).

8. Ast, C. R. et al. Giant spin splitting through surface alloying. Phys. Rev. Lett. 98, 186807 (2007).

9. Ast, C. R. et al. Local detection of spin-orbit splitting by scanning tunneling spectroscopy. Phys. Rev. B 75, 201401(R) (2007).

10. Kato, Y. K., Myers, R. C., Gossard, A. C. \& Awschalom, D. D. Observation of the spin Hall effect in semiconductors. Science 306, 1910-1913 (2004).

11. Mills, A. P. Jr. Positronium formation at surfaces. Phys. Rev. Lett. 41, 1828-1831 (1978).

12. Gidley, D. W., Köymen, A. R. \& Capehart, T. W. Polarized low-energy positrons: A new probe of surface magnetism. Phys. Rev. Lett. 49, 1779-1783 (1982).

13. Kawasuso, A. et al. Current-induced spin polarization on a Pt surface: A new approach using spin-poalrized positron annihilation spectroscopy. J. Magn. Magn. Mater. 342, 139-143 (2013).

14. Kawasuso, A. \& Maekawa, M. Spin-polarization of an electro-static positron beam. Appl. Surf. Sci. 255, 108-110 (2008).

15. Jiang, A. et al. The structure and stability of $\beta$-Ta thin films. Thin solid films 479 , 166-173 (2005).

16. Weerasekera, I. A., Shah, S. I., Baxter, D. V. \& Unruh, K. M. Structure and stability of sputter deposited beta-tungsten thin films. Appl. Phys. Lett. 64, 3231-3233 (1994).

17. Karabacak, T., Wang, P. I., Wang, G. C. \& Lu, T. M. Phase transformation of single crystal $\beta$-tungsten nanorods at elevated temperatures. Thin solid films 493, 293-296 (2005).

18. Coleman, P. Positron Beams and their Applications (World Scientific, Singapore, 2000).

19. Chu, S., Mills, A. P. Jr. \& Murray, C. A. Thermodynamics of positronium thermal desorption from surfaces. Phys. Rev. B 23, 2060-2064 (1981).

20. Rosenberg, I. J., Howell, R. H. \& Fluss, M. J. Temperature dependence of the positronium work function. Phys. Rev. B 35, 2083-2086 (1987).

21. Michaelson, H. B. The work function of the elements and its periodicity. J. Appl. Phys. 48, 4729-4733 (1977).

22. Kimura, T., Otani, Y., Sato, T., Takahashi, S. \& Maekawa, S. Room-temperature reversible spin Hall effect. Phys. Rev. Lett. 98, 156601 (2007).

23. Ando, K. et al. Electric manipulation of spin relaxation using the spin Hall effect. Phys. Rev. Lett. 101, 036601 (2008).

24. Mosendz, O. et al. Detection and quantification of inverse spin Hall effect from spin pumping in permalloy/normal metal bilayers. Phys. Rev. B 82, 214403 (2010).

25. Liu, L. Q., Moriyama, T., Ralph, D. C. \& Buhrman, R. A. Spin-torque ferromagnetic resonance induced by the spin Hall effect. Phys. Rev. Lett. 106, 036601 (2011).

26. Morota, M. et al. Indication of intrinsic spin Hall effect in $4 \mathrm{~d}$ and $5 \mathrm{~d}$ transition metals. Phys. Rev. B 83, 174405 (2011).
27. Kondou, K., Sukegawa, H., Mitani, S., Tsukagoshi, K. \& Kaisai, S. Evaluation of spin Hall angle and spin diffusion length by using spin current-induced ferromagnetic resonance. Appl. Phys. Exp. 5, 073002 (2012).

28. Hahn, C. et al. Comparative measurements of inverse spin Hall effects and magnetoresistance in YIG/Pt and YIG/Ta. Phys. Rev. B 87, 174417 (2013).

29. Althammer, M. et al. Quantitative study of the spin Hall magnetoresistance in ferromagnetic insulator/normal metal hybrids. Phys. Rev. B 87, 224401 (2013).

30. Tanaka, T. et al. Intrinsic spin Hall effect and orbital Hall effect in $4 \mathrm{~d}$ and $5 \mathrm{~d}$ transition metals. Phys. Rev. B 77, 165117 (2008).

31. Kontani, H., Tanaka, T., Hirashima, D. S., Yamada, K. \& Inoue, J. Giant orbital Hall effect in transition metals: Origin of large spin and anomalous Hall effects. Phys. Rev. Lett. 102, 016601 (2009).

32. Zhang, S. F. Spin effect in the presence of spin diffusion. Phys. Rev. Lett. 85, 393-396 (2000).

33. Edelstein, V. M. Spin polarizaton of conduction electrons induced by electric current in twodimensional asymmetric electron systems. Sol. Stat. Commun. 73, 233-235 (1990).

34. Gambardella, P. \& Miron, I. M. Current-induced spin-orbit torques. Philos. Trans. A Math. Phys. Eng. Sci. 369, 3175-3197 (2011).

35. Rojas Sánchez, J. C. et al. Spin-to-charge conversion using Rashba coupling at the interface between non-magnetic materials. Nat. commun. 4, 2944 (2013).

36. Schwab, P., Raimondi, R. \& Gorini, C. Inverse spin Hall effect and anomalous Hall effect in a two-dimensional electron gas. EPL 90, 67004 (2010).

37. Liu, X. et al. Structure and magnetization of small monodisperse platinum clusters. Phys. Rev. Lett. 97, 253401 (2006).

38. Sampedro, B. et al. Ferromagnetism in fcc twinned $2.4 \mathrm{~nm}$ size Pd nanoparticles. Phys. Rev. Lett. 91, 237203 (2003).

39. Shimizu, S. et al. Eletrically tunable anomalous Hall effect in Pt thin films. Phys. Rev. Lett. 111, 216803 (2013).

40. Lynn, K. G. \& Welch, D. O. Slow positrons in metal single crystals. I. Positronium formation at $\mathrm{Ag}(100), \operatorname{Ag}(111)$, and $\mathrm{Cu}(111)$ surfaces. Phys. Rev. B 22, 99-110 (1980).

\section{Acknowledgments}

We are grateful to B. Gu, J. Ieda, and S. Maekawa of Japan Atomic Energy Agency (JAEA) for their valuable suggestions and discussions. This work was financially supported by JSPS KAKENHI Grant No. 24310072 and a Grant for Basic Science Research Projects from the Sumitomo Foundation.

\section{Author contributions}

A.K. constructed the spin-polarized positron beam, A.K., Y.F., M.M., and H.J.Z. constructed the CISP measurement system. H.J.Z. proposed the project. E.S., K.T., T.S., H.J.Z. and S.Y. prepared the samples, H.J.Z. and H.L. performed the CISP experiments, S.Y performed the XRD experiments. H.J.Z. performed the data analysis and wrote the manuscript, all authors discussed the results and revised the manuscript.

\section{Additional information}

Competing financial interests: The authors declare no competing financial interests. How to cite this article: Zhang, H.J. et al. Current-induced spin polarization on metal surfaces probed by spin-polarized positron beam. Sci. Rep. 4, 4844; DOI:10.1038/srep04844 (2014).

(i) This work is licensed under a Creative Commons Attribution 3.0 Unported License The images in this article are included in the article's Creative Commons license, unless indicated otherwise in the image credit; if the image is not included under the Creative Commons license, users will need to obtain permission from the license holder in order to reproduce the image. To view a copy of this license, visit http://creativecommons.org/licenses/by/3.0/ 